\title{
The Role of Flavor Physics in the LHC Era
}

\author{
Stephen Lars Olsen*it: \\ Seoul National University \\ E-mail: solsen@hep1.snu.ac.kr
}

\begin{abstract}
Although searches for new physics at the CERN Large Hadron Collider will probably dominate the the agenda of the experimental high energy physics community during the next decade or more, high-intensity experiments at the $\tau$-charm and beauty thresholds will continue to play important complementary roles. These include the establishment of stringent constraints on proposed theories for beyond-the-Standard-Model physics and unique opportunities to address some new physics scenarios that are inaccessible at the LHC. In addition, in the event that the LHC does discover some new phenomena, high sensitivity flavor physics measurements could provide diagnostic clues as to the physics processes responsible for the observed effects. In this talk I present a few examples that illustrate the close inter-relation of new physics searches at the high-energy frontier and high-sesitivity measuremenst at the intensity frontier.
\end{abstract}

52 International Winter Meeting on Nuclear Physics - Bormio 2014,

27-31 January 2014

Bormio, Italy

\footnotetext{
* Speaker.

$\dagger$ Supported by the Korean National Research Foundation (NRF) Grant No. 20110029457.

$\ddagger$ Current address: Institute of Basic Science, Daejeon, Korea
} 


\section{Introduction}

The discovery of the Higgs boson in 2012 by the ATLAS [1] and CMS [2] experiments at the CERN Large Hadron Collider (LHC) provided the long-awaited experiment evidence that "completed" the Sandard Model (SM). Although this model has been phenomenally successful at reproducing the results of all particle physics experiments that have been reported to date, it has a large number of parameters that have to be input from experiment, it does not provide any explanation for dark matter, dark energy or the matter-antimatter asymmetry of the Universe, and does not incorporate Gravity. For these and other reasons, most particle physics researchers believe that the SM, in spite of its considerable success, is not a complete theory of nature.

As a result, a number of extensions of the SM, so-called beyond-the-SM (BSM) theories, have been proposed, most of which predict the existence of new, as yet unseen, massive particles [3], usually with masses in the $100 \mathrm{GeV} \sim 2 \mathrm{TeV}$ range that is accessible at the LHC. The most commonly discussed BSM theory is Supersymmetry (SUSY), which proposes a SUSY partner for each of the established SM partners, and an assortment of five Higgs particles that include a doublet of charged Higgs scalars [4]. Other BSM theories predict heavy versions of the $Z$ and $W$ bosons [5], a fourth-generation of quarks [6], etc. To the disappointment of many practitioners in the field, the first operational period of the LHC, which studied $p p$ collisions in the $\sqrt{s}=7 \sim 8 \mathrm{TeV}$ range, uncovered no new particles (besides the Higgs), and most of the SUSY parameter space for SUSY partner particle with masses below $M \sim 1 \mathrm{TeV}$ has been ruled out [7]. However, the LHC is now in the process of (approximately) doubling both the CM energy and the luminosity, and hopes remain high that first signs of BSM particles will emerge in the higher energy data that will start becoming available in early 2015. It can be expected that the LHC and the ATLAS and CMS energy frontier, high- $p_{T}$, high luminosity experiments will remain the "Flagship" experimental high energy particle physics programs for at least the next decade.

In contrast to ATLAS and CMS, the LHCb experiment runs at lower luminosity (a few $\mathrm{fb}^{-1} / \mathrm{yr}$ ) and exploits the large cross section for $B$ meson production in multi-TeV $p p$ collisions $(\simeq 300 \mu \mathrm{b}$ at $\sqrt{s}=7 \mathrm{TeV}$ ) to do high precision measurements of the decay properties of particles containing $b$ - and/or $c$-quarks. The $\mathrm{LHCb}$ experiment has been remarkably productive in a number of areas. It has recently made first observation (with $4 \sigma$ statistical significance) of the very rare decay $B_{s} \rightarrow$ $\mu^{+} \mu^{-}$process $\left(\mathscr{B}\left(B_{s} \rightarrow \mu^{+} \mu^{-} \simeq 3 \times 10^{-9}\right.\right.$ !) $[8,9]$ and made spectacular measurements of the $B_{s}-\bar{B}_{s}$ mixing frequency with an impressive $0.1 \%$-level precision: $f=\Delta m_{s}=17.768 \pm 0.0 .23 \pm$ $0.006 \mathrm{ps}^{-1}[10,9]$. As discussed at this meeting by U. Uwer [9], these and other measurements place strong constraints on a number of proposed BSM theories [11].

Prior to the startup of $\mathrm{LHCb}$, the BaBar and Belle B-factory experiments dominated the landscape of $B$ and $D$ meson decay physics. The main goals of these experiments were tests of the $\mathrm{SM}$ mechanism for $\mathrm{CP}$ violation [12]. Here the highlight of both groups' research programs in the first half of the 2000-2009 decade were measurements of the $C P$ violating phase $\phi_{1}$ (aka $\beta$ ) in time-dependent $C P V$ asymmetries in $B^{0}$ meson decays into $C P$ eigenstates such as $K^{0} J / \psi$. Their measurements [13] confirmed SM expectations and led to Nobel prizes for Kobayashi and Maskawa in 2008. Subsequent notable results from these experiments, which placed important constraints on BSM theories, included measurements of time-dependent $C P$ violating phases in penguin dominated $C P$ eigenstate decay modes such as $B^{0} \rightarrow K^{0} \phi$ and $B^{0} \rightarrow K^{0} \eta^{\prime}$ [14] and measurements of 
$B$ mesons decays to final states containing $\tau$ leptons, i.e., purely leptonic $B^{+} \rightarrow \tau^{+} v_{\tau}[15,16]$ and semileptonic $B \rightarrow D^{(*)} \tau^{+} v_{\tau}[17,18]$ decays.

BaBar stopped taking data in 2008 by which time it had accumulated a data sample that corresponded to a total integrated luminosity of $531 \mathrm{fb}^{-1}$; Belle stopped in 2010 after accumulating a $1040 \mathrm{fb}^{-1}$ data sample. The KEKB collider and the Belle detector are currently being upgraded to SuperKEKB and BelleII. The SuperKEKB design luminosity is 40 times that of KEKB and will start providing yearly data samples of $10 \mathrm{ab}^{-1}$ by about $2019\left(1 \mathrm{ab}^{-1}=1000 \mathrm{fb}^{1}\right)$. The LHCb and BelleII programs will be largely complementary: the yearly samples of $B$ mesons registered by LHCb and BelleII will be similar. Because of the larger boost factors, LHCb will have much better vertexing than BelleII and they will accumulate $B$ and $B_{s}$ decays simultaneously. On the other hand, thanks to the clean $e^{+} e^{-} \rightarrow \Upsilon(4 S) \rightarrow B \bar{B}$ environment, BelleII will be uniquely capable of studying inclusive modes such as $b \rightarrow s \gamma$ and $b \rightarrow u \gamma$, and modes with missing energy, such as $B^{+} \rightarrow \tau^{-} v_{\tau}$. However, for $B_{s}$ physics, BelleII would have to run at the $\Upsilon(5 S)$, where the production cross section for $B_{s}$ and $B$ meson production is about a factor of five lower.

In this report I will give a few examples of how intensity-frontier flavor physics measurements impact BSM searches at the energy-frontier. For more comprehensive discussions I refer the reader to Refs. [19] and [20].

\section{Particle-antiparticle mixing}

The idea that neutral $K^{0}$ mesons would spontaneously change into their antiparticle $\bar{K}^{0}$ (and vice versa) was first proposed by Gell Mann and Pais in 1955 [21]. Measurements of the properties of the related $K_{S}$ and $K_{L}$ eigenstates was a major activity in the 1960s and led to the discovery of $C P$ violation in 1964 [22]. In the SM, the frequency for $K^{0}-\bar{K}^{0}$ mixing or, equivalently, the $K_{S}-K_{L}$ mass difference $\Delta m_{K}$, is adequately described by the imaginary part of the four-quark process shown in Figure 1.
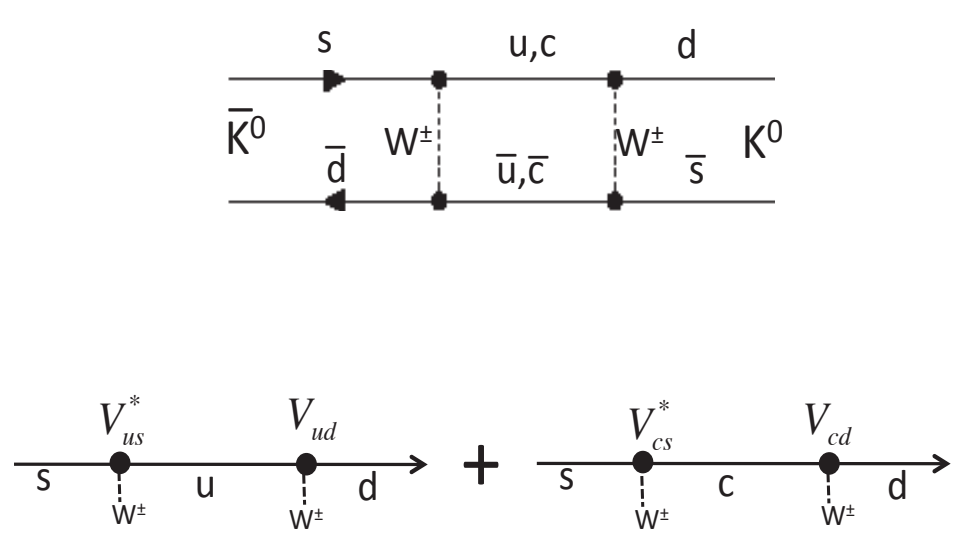

Figure 1: One of the quark-level box diagrams responsible for $K^{0}-\bar{K}^{0}$ mixing (upper). Illustration of the $\Delta m_{K}$ calculation (lower).

From the lower portion of Fig. 1, we can see that

$$
\Delta m_{K} \propto G_{F}^{2}\left(V_{u s}^{*} V_{u d} f\left(m_{u}\right)+V_{c s}^{*} V_{c d} f\left(m_{c}\right)\right) .
$$


In a four-quark world, the quark-flavor mixing matrix is simply a two-dimensional rotation by the Cabibbo angle $\left(\theta_{C}\right)$ :

$$
\left(\begin{array}{cc}
V_{u d} & V_{u s} \\
V_{c d} & V_{c s}
\end{array}\right)=\left(\begin{array}{cc}
\cos \theta_{C} & \sin \theta_{C} \\
-\sin \theta_{C} & \cos \theta_{C}
\end{array}\right),
$$

and Eq. 2.1 becomes

$$
\Delta m_{K}^{\mathrm{SM}} \propto G_{F}^{2}\left(f\left(m_{u}\right)-f\left(m_{c}\right)\right) \cos \theta_{C} \sin \theta_{C} \simeq G_{F}^{2} m_{c}^{2} \cos \theta_{C} \sin \theta_{C} .
$$

The mixing frequency depends on the difference beiween the $c$ - and $u$-quark masses, in fact, almost entirely on the $c$-quark mass. Thus, when Glashow, Illiopulis and Maiani proposed the existence of the charmed quark in 1970 [23], they predicted its mass to be "not larger than $3 \sim 4 \mathrm{GeV}$," based on the measured value of $\Delta m_{K}$ at that time.
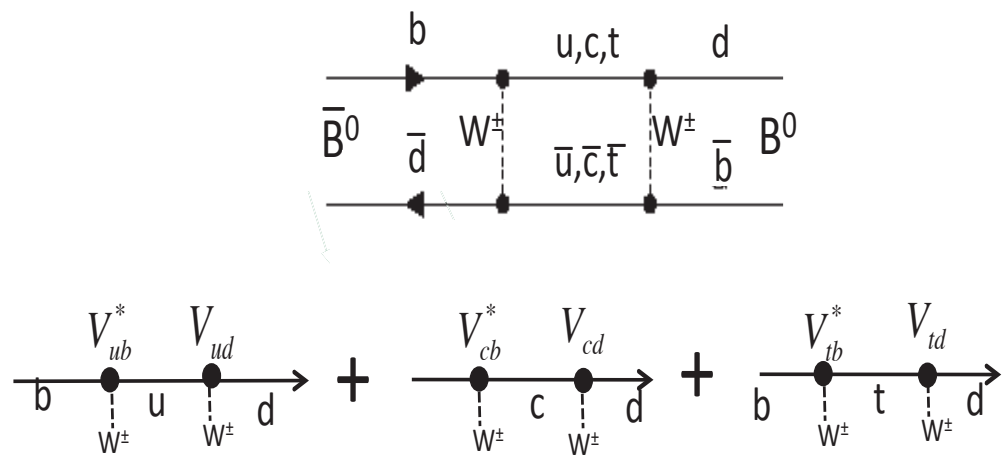

Figure 2: One of the quark-level box diagrams responsible for $B^{0}-\bar{B}^{0}$ mixing (upper) and an illustration of the $\Delta m_{d}$ calculation (lower).

The major flavor physics event of of the 1980s was the unexpected discovery of $B^{0}-\bar{B}^{0}$ mixing by the ARGUS experiment in 1987 [24]. In this case, since the mixing involved the third-generation $b$-quark, a six-quark analysis, as indicated in Fig. 2, is necessary. The expression for the neutral $B$ meson eigenstate mass difference $\Delta m_{d}$ is

$$
\Delta m_{d}^{\mathrm{SM}} \propto G_{F}^{2}\left(V_{u b}^{*} V_{u d} f\left(m_{u}\right)+V_{c b}^{*} V_{c d} f\left(m_{c}\right)+V_{t b}^{*} V_{t d} f\left(m_{t}\right)\right),
$$

where $V_{i j}$ is the $i j^{\text {th }}$ element of the well known CKM six-quark-flavor mixing-matrix. Note that if the quark masses were degenerate, i.e., $m_{u}=m_{c}=m_{t}, \Delta m_{d}$ would be proportional to $V_{u b}^{*} V_{u d}+$ $V_{c b}^{*} V_{c d}+V_{t b}^{*} V_{t d}$, which the unitarity of the CKM guarantees to be zero. So, in this case also, the $B^{0}-\bar{B}^{0}$ mixing frequency depends on the non-degeneracy of the quark masses and, to a good approximation depends primarily on the top-quark mass. The SM expectation is

$$
\Delta m_{d}^{\mathrm{SM}} \propto G_{F}^{2} m_{t}^{2}\left|V_{t b}^{*} V_{t d}\right|^{2} .
$$

Thus, as a consequence of the ARGUS discovery of large $B^{\triangleleft 0}-\bar{B}^{0}$ mixing, it was realized that the top-quark mass was much higher than was previously thought to be the case. ${ }^{1}$ Specific calculations gave values around $m_{t} \simeq 170 \mathrm{GeV}$ [25]. The top quark was discovered in 1995 [26] and its mass is measured to be $173.5 \mathrm{GeV}$ with $\simeq 0.3 \%$ precision [27].

\footnotetext{
${ }^{1}$ Much to the dismay of those of us who, at the time, were searching for the $t$-quark in the $m_{t} \simeq 30 \mathrm{GeV}$ mass region.
} 


\subsection{Influence of SUSY on particle-antiparticle mixing}

In the SM, there are no Flavor Changing Neutral Currents $(F C N C)$ that directly convert $s \rightarrow d$ or $b \rightarrow s$, etc. Thus, the SM descripton for mixing necessarily involves second-order weak-interaction box diagrams as shown in Figs. 1 and 2 above. The process is mediated by heavy virtual particles: in the case of $B^{0}-\bar{B}^{0}$ mixing, by virtual top-quarks and $W$-bosons. If SUSY exists, virtual SUSY partner particles could also occur as virtual legs in the mixing box diagrams and, thus, cause differences between the measured mixing frequencies and their SM prediction.

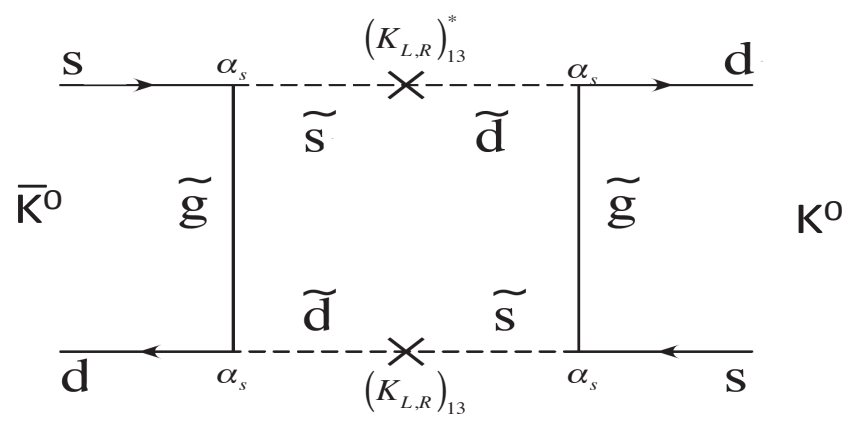

Figure 3: A potential SUSY box diagram contribution to $K^{0}-\bar{K}^{0}$ mixing. Here the vertical lines represent gluino propagators and the dashed lines squark propagators.

A typical SUSY contribution to mixing is shown in Fig. 3, with virtual squarks and gluinos replacing the $W \mathrm{~s}$ and up-type quarks in the SM diagrams shown in Figs. 1 and 2. Here the $\left(K_{L, R}\right)_{13}^{*}$ terms denote off-diagonal elements of a mixing matrix that accounts for the possibility that squark flavors are not necessarily aligned with ordinary weak interaction flavors. Here, unlike the SM diagrams, the vertices at the corners of the box are not due to weak interactions, but are strong interactions. Thus, instead of being proportional the weak coupling $G_{F}$, they are proportional to the QCD coupling $\alpha_{s}$, which is much larger. Thus, potential SUSY contributions to ordinary particle-antiparticle mixing are, a prior, large.

Grossman provided a convenient formula that characterizes the relative strength of the SUSY and SM contributions to $\Delta m_{K}[28]$ :

$$
\frac{\Delta m_{K}^{\mathrm{SUSY}}}{\Delta m_{K}^{\mathrm{SM}}} \sim 10^{4}\left(\frac{100 \mathrm{GeV}}{m_{\tilde{Q}}}\right)^{2}\left(\frac{\Delta m_{\tilde{Q}}^{2}}{m_{\tilde{Q}}^{2}}\right)^{2} \mathscr{R} e\left[\left(K_{L}\right)_{13}\left(K_{R}\right)_{13}\right] .
$$

Here $m_{\tilde{Q}}$ is the squark mass and $\Delta m_{\tilde{Q}}$ is some measure of squark mass differences.

Table 1: Measured particle-antiparticle mixing frequencies and their SM expected values.

\begin{tabular}{c|c|c}
\hline Channel & $\begin{array}{c}\Delta m \text { (expt) } \\
\left(\mathrm{ps}^{-1}\right)\end{array}$ & $\begin{array}{c}\Delta m \text { (theory) } \\
\left(\mathrm{ps}^{-1}\right)\end{array}$ \\
\hline \hline$K^{0}-\bar{K}^{0}$ & $0.00530 \pm 0.00001$ & $0.00502 \pm 0.00051$ \\
$B^{0}-\bar{B}^{0}$ & $0.510 \pm 0.004$ & $0.55_{-0.05}^{+0.07}$ \\
$B_{s}^{0}-\bar{B}_{s}^{0}$ & $17.69 \pm 0.08$ & $17.3 \pm 2.6$ \\
\hline \hline
\end{tabular}


The measured values of the mixing frequencies for $K^{0}, B^{0}$ and $B_{s}^{0}$, taken from the HFAG [29] and PDG [27] averages, are compared to theoretical expectations [30, 31] in Table 1. In all cases there is good agreement; the comparison is limited by the precision of the theoretical calculations, which have errors in all three cases that are about $\pm 10 \%$. The does not leave a lot of room for SUSY contributions per Eq. 2.6. If we, somewhat arbitarily, assume that the $10 \%$-level of agreement between experiment and SM predictions given in Table 1 constrains the ratio in Eq. 2.6 to be less than 0.3, we find that for a squark mass that would have been accessible at the recent LHC run, i.e. $m_{\tilde{Q}}<1 \mathrm{TeV}$,

$$
\left(\frac{\Delta m_{\tilde{Q}}^{2}}{m_{\tilde{Q}}^{2}}\right)^{2} \mathscr{R} e\left[\left(K_{L}\right)_{13}\left(K_{R}\right)_{13}\right]<\sim 0.003 .
$$

This says that the venerable, 50 year-old, measurements of $\Delta m_{K}$ place very severe constraints on SUSY. Either the squark masses must be highly degenerate, and very unlike the quark masses which range over nearly five orders of magnitude, or the SUSY squark flavors must align with the weak interaction quark flavors to an extraordinary degree of precision, or some combination of the two. Since there is nothing in the SUSY theory that would naturally enforce such restrictions, this is called "the Flavor Problem," which is well known inside the SUSY community, but less well know generally.

To address this problem in a "natural" way, Nir and Raz devised a symmetry principle that restricted the off-diagonal $\left(K_{L, R}\right)$ matricx elements to higher order values of $\sin \theta_{C}$, thereby limiting down-type squark contributions to particle-antiparticle mixing to acceptable values. However, this so-called "horizontal symmetry" necessarily requires up-type squark matrix elements to be of order $\sin \theta_{C}$, which would make large SUSY contributions to the $D^{0}-\bar{D}^{0}$ mixing frequency $\left(\Delta m_{c}\right)$; they predicted $\Delta m_{c} \simeq 0.1 \mathrm{ps}^{-1}$ [32]. Subsequent to the Nir-Raz paper, anomalously large $D^{0}-\bar{D}^{0}$ mixing was observed by Belle and BaBar [33], however this appears to be mostly in the real part of the mixing amplitude that relates the life-time difference between the neutral $D$-meson eigenstates. The HFAG group's average of the mass-difference measurements are within $\sim 2 \sigma$ of zero [29], the $95 \%$ confidence level upper limit is $\Delta m_{c}<0.01 \mathrm{ps}^{-1}$, an order of magnitude below the Nir-Raz prediction.

\subsection{Time-dependent $C P$ violation asymmeties}

The experimental precision of the mixing measurements discussed in the previous section far exceeds that of the predictions based on SM theory. Until the theoretical precision is improved, there is no pressing need for more precise experimental measurements. However, the situation is reversed for the case of measurements of $C P$ violating phases in mixing-induced interference effects. For these, since $\mathrm{QCD}$ is $C P$ conserving, SM predictions for the $C P$ violating phases are not effected by long-range effects or additional gluons etc., and are consequently less ambiguous and more precise.

Mixing-induced time-dependent $C P$ violating asymmetries are due to the interference between the direct decay and of a $B^{0}$ meson to a $C P$ eigenstate such as $B^{0} \rightarrow K^{0} J / \psi$ and the process where tthe $B^{0}$ first transforms into a $\bar{B}^{0}$ and then decays to the same final state: $B^{0} \rightarrow \bar{B}^{0} \rightarrow K^{0} J / \psi$, as illustrated in Fig. 4. Here the $t \rightarrow d$ vertices, which have a strength proportional to $V_{t d}^{*}$, have a $C P$ violating complex phase $\phi_{1}$. In the SM, all the other vertices in the diagrams shown in Fig. 4 are 
real. The interference between the two diagrams is proportional to $V_{t d}^{*} V_{t d}^{*}$ and has a CPV phase of $2 \phi_{1}$.

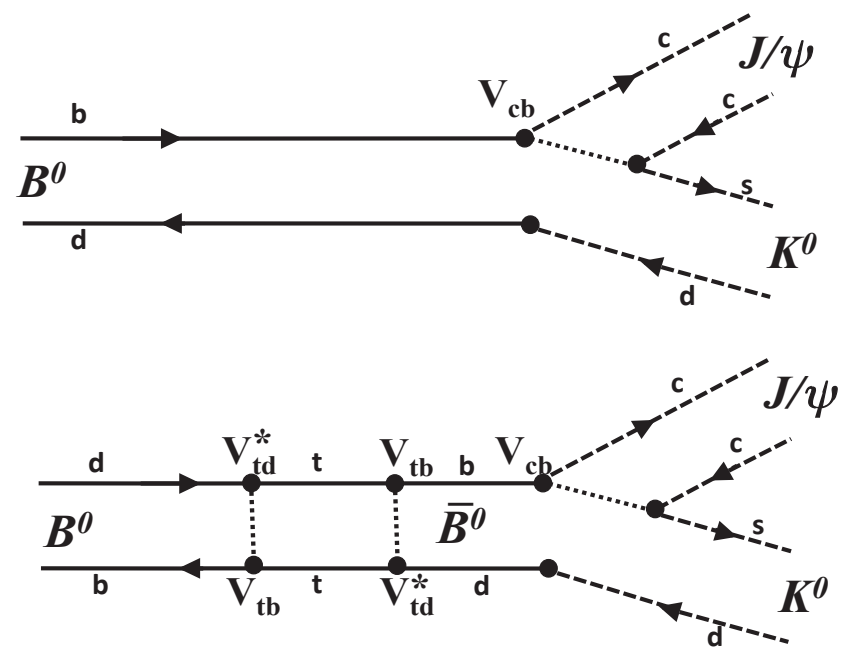

Figure 4: Quark flow diagrams that produce mixing-induced $C P$ violations. The direct decay $B^{0} \rightarrow K^{0} J / \psi($ upper $)$ interferes with $B^{0} \rightarrow \bar{B}^{0} \rightarrow K^{0} J / \psi$ decays (lower). Here the only flavor changing vertices with a non-zero $C P V$ phase is the $t \rightarrow d$ transition. The interference amplitude is proportional to $\sin 2 \phi_{1}$, where $\phi_{1}$ is the phase of $V_{t d}$.

The measurement technique used at the $B$-factories is illustrated in Fig. 5. A coherent $B^{0} \bar{B}^{0}$ meson pair is produced in an asymmetric $e^{+} e^{-}$collision. After some time, one of the mesons $\left(B_{\mathrm{tag}}\right)$ decays into a flavor-specific final state, i.e., a final state that reflects the $B$-flavor of the decaying meson. (These types of decays occur more than $90 \%$ of the time.) If the flavor of $B_{\text {tag }}$ can be tagged, e.g. by the charge of a lepton from semileptonic decay or a charged kaon from the $b \rightarrow c \rightarrow s$ decay sequence, that ensures that the accompanying meson, $B_{C P}$, has the opposite $B$-flavor at that time, which is set as $t=0$. Then, as $B_{C P}$ evolves with time, it starts to mix into its antiparticle state, the unmixed and mixed components of $B_{C P}$ interfere. The interference can be seen when $B_{C P}$ decays into a $C P$ eigenstate. The asymmetry between the number of times the $B_{\text {tag }}$ decay was a $B^{0}$ or a $\bar{B}^{0}$ as a function of the time that $B_{C P}$ decays, follows a $\sin \Delta m_{d} t$ curve (both forward and backward in time) with amplitude $\sin 2 \phi_{1}$, as sketched in the figure. The relative time between the two decays is inferred from the measured $z$ position of each decay vertex. Figure 5 shows the case for the $C P=-1, B_{C P} \rightarrow K_{S} J / \psi$ decay mode. Decays to $C P=+1$ final states, such as $B_{C P} \rightarrow K_{L} J / \psi$, have the opposite asymmetry. In the actual experiment, the amplitude of the asymmetry curve is reduced from its ideal value of $\sin 2 \phi_{1}$ by experimental dilution factors, primarily due to incorrectly tagged $B_{\text {tag }}$ decays. The effects of these mistags are measured with high statistics $B^{0}$ semileptonic decay events in the same data samples and are experimentally well understood.

This technique has been carefully developed and optimized by both the Belle and BaBar teams and it is now quite well understood. Results based on the full data samples from the two groups [34] are shown in Fig. 6. In these figures results from the $C P=-1$ and $C P=+1$ final states are shown separately and they display opposite-sign asymmetries, as expected. The measurements of $\phi_{1}$ are quite precise; the current average value of the two groups' measurements is $\sin 2 \phi_{1}=0.679 \pm 0.020$ (corresponding to $\phi_{1}=(21.38 \pm 0.79)^{\circ}$ ). In both cases, the precision is still limited by statistics. 


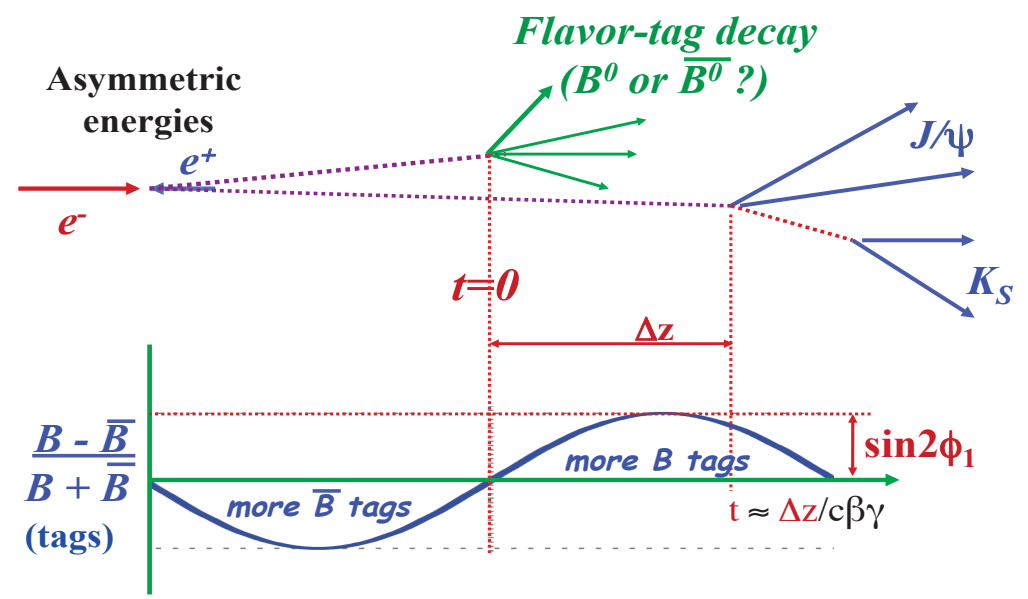

Figure 5: An illustration of the technique used to measure mixing-induced time-dependent $C P V$ asymmetries at the $B$-factories.

It is expected that the precision could be improved by about a factor of two before measurements using this technique are systematics limited. This is about the level of validity of the theoretical equivalence of the measured asymmetry and $\sin 2 \phi_{1}$.
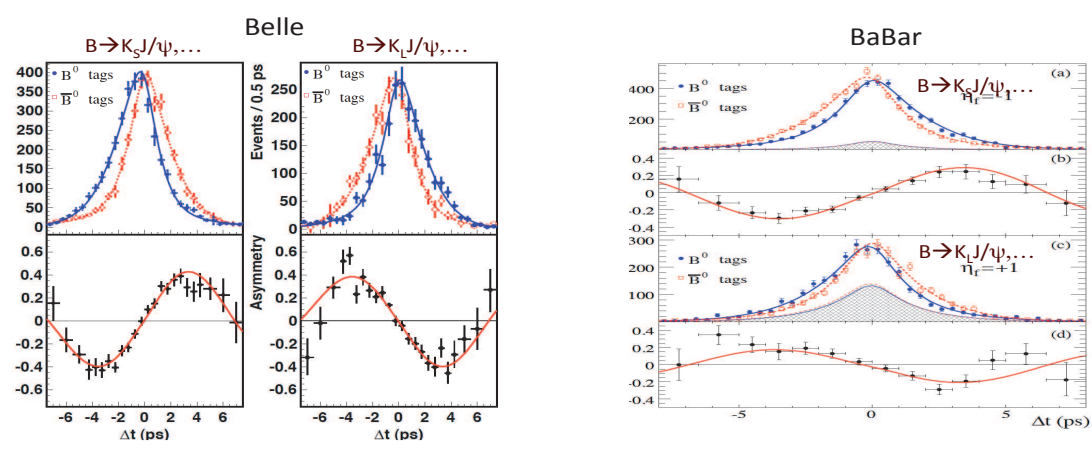

Figure 6: Belle (left) and BaBar (right) measurements of $C P$ violations for $B^{0} \rightarrow K^{0}(c \bar{c})$ decays. The upper plots show the time distributions for numbers of $B^{0}$ - and $\bar{B}^{0}$-tags and the lower plots show their time-dependent asymmetries.

\subsection{BSM searches using mixing-induced $C P$-violating asymmetry measurements.}

Sensitive searches for signs of new, BSM physics can be made by applying the above-described mixing-induced $C P V$ asymmetry measurement technique to rare $B^{0} \rightarrow C P$-eigenstate decays that are mediated by penguin diagrams. An example is the $B^{0} \rightarrow K_{S} \phi$ decay mode, for which the SM leading order decay amplitude is the penguin-mediated process shown in Fig. 7(a). The CKM matrix elements involved here are $V_{t b}^{*}$ and $V_{t s}$, neither of which have a $C P V$ phase. Thus, as in the case of $B^{0} \rightarrow K^{0} J / \psi$ etc., the interference between the direct $B^{0} \rightarrow K_{S} \phi$ decay amplitude and the mixed $B^{0} \rightarrow \bar{B}^{0} \rightarrow K_{S} \phi$ amplitude all comes from mixing and will have the same $2 \phi_{1} C P V$ phase, and the interference asymmetry will have the same $\sin 2 \phi_{1}$ amplitude.

However, BSM theories that have new particles that can couple to $b$ - and $s$-quarks can, in principle, modify this process. For example, in the case of SUSY, the $W$ and $t$-quark in the SM 
process could be replaced by a squark and a chargino (the SUSY partner of the $W$ ), as shown in Fig. 7(b). In that case, the SUSY part of the decay amplitude would contribute a different $C P V$ phase. (SUSY has 44 non-trivial $C P V$ phases.) Thus, a significant difference between the mixinginduced $C P$-violating asymmetry in $B^{0} \rightarrow K_{S} \phi\left(\sin 2 \phi_{1}^{\text {eff }}\right)$ and that in $B^{0} \rightarrow K_{S} J / \psi$ would be a clear sign of new, BSM physics. This is also the case for many other penguin-mediated rare decays, such as $B^{0} \rightarrow K_{S} \eta^{\prime}, B^{0} \rightarrow K_{S} \pi^{0}$, etc.

a)

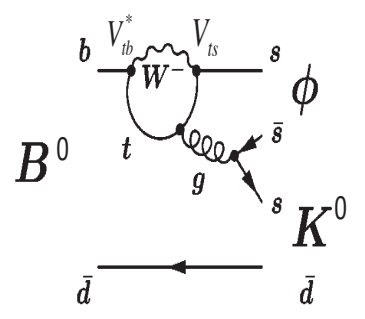

b)

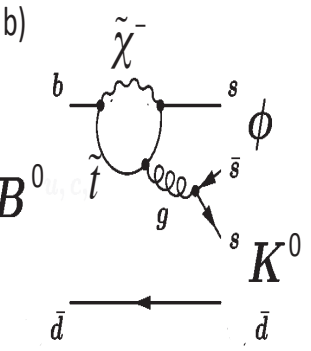

$B \rightarrow K_{S} \phi$

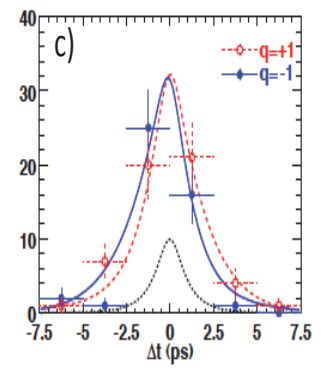

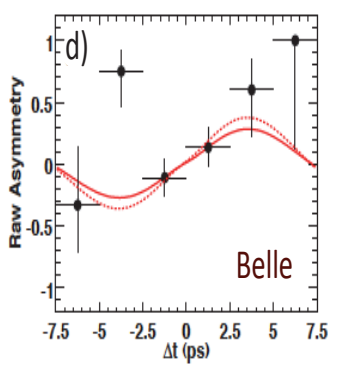

Figure 7: $a$ ) The SM penguin diagram for $B^{0} \rightarrow K^{0} \phi$ decays and $b$ ) possible SUSY contributions. Belle measurements of the time-dependent $B^{0}$ and $\bar{B}^{0}$-tag distributions and the $C P V$ asymmetry for $B^{0} \rightarrow K_{S} J / \psi$ decays are shown in $c$ ) and d), respectively.

Such measurements were carried out by Belle and BaBar [14]; results from the Belle measurement are shown in Figs. 7(c) and (d). The weighted average of the two groups' measurements of $\sin 2 \phi_{1}^{\text {eff }}$ for $B^{0} \rightarrow K_{s} \phi$ decays is $\sin 2 \phi_{1}^{\text {eff }}=0.39 \pm 0.17$, which is $1.8 \sigma$ below the SM expectation (of $0.679 \pm 0.020$ ). Results for other penguin processes have similar precision. The main current issue is statistics. Penguin processes are rare: the branching fraction for $B^{0} \rightarrow K_{S} \phi$ is a factor of a hundred smaller than that for $B^{0} \rightarrow K_{S} J / \psi$. Thus, while the Belle measurements shown in Fig. 6 include over $12 \mathrm{~K} B \rightarrow K_{S} J / \psi$ events and more than $10 \mathrm{~K} B \rightarrow K_{L} J / \psi$ events, the $K_{s} \phi$ measurements shown in Fig. 7 are based on only about $150 B \rightarrow K_{S} \phi$ events. BelleII ultimately expects to accumulate a data sample that is $\sim 40$ times larger than the Belle data sample. With such a sample, $\sin 2 \phi_{1}^{\text {eff }}$ will be measured for $K_{S} \phi$ and many other penguin modes with a precisions of $\sim \pm 0.03$ for each mode. These will severely test the SM.

The LHCb experiment is also challenging the SM with measurements of the mixing-induced phase $\phi_{s}$ in $B_{s} \rightarrow \phi J / \psi$ decays. Since none of the CKM elements in the SM box diagram for $B_{s}^{0} \rightarrow \bar{B}_{s}^{0}$ have a $C P V$ phase, the SM prediction for $\phi_{s}$ is that it should be very small. This was discussed at this meeting by U. Uwer [9].

\subsection{Generic new physics limits from mixing-induced $C P$-violation measurements}

From dimensional analysis alone, the inclusion of new, BSM physics at a high mass scale $\Lambda_{i}$ that mediates the Flavor Changing Neutral Currents $(s \rightarrow d, b \rightarrow d b \rightarrow s$ and $c \rightarrow u)$ that are at play in particle-antiparticle mixing could be described by an effective Lagrangian of the form

$$
\mathscr{L}_{\text {eff }}=\mathscr{L}_{\mathrm{SM}}+\frac{c_{i}}{\Lambda_{i}^{2}} O_{i}(F C N C),
$$

where $c_{i}$ is the coupling strength that depends on the details of the new physics theory, and $O_{i}(F C N C)$ is the operator that produces the transition. Thus, the influence of new physics effects 
depends on $c_{i} / \Lambda_{1}^{2}$. Figure 8 illustrates the limits that current particle-antiparticle mixing measurements place on $\Lambda_{i}$ for the cases where $c_{i}=1$ [35]. For the $i=s \rightarrow d$ transition, the allowed mass scales are all above $10^{3} \mathrm{TeV}$. For the other channels, the limits start nearer $10^{2} \mathrm{TeV}$, but still are quite impressive. Thus, as we noticed in the discussion associated with Eq. 2.7 above, for new physics with mass scales that are accessible at current or future runs of the LHC, the theories are tightly constrained to have very, very small values for the $c_{i}$ coefficients.

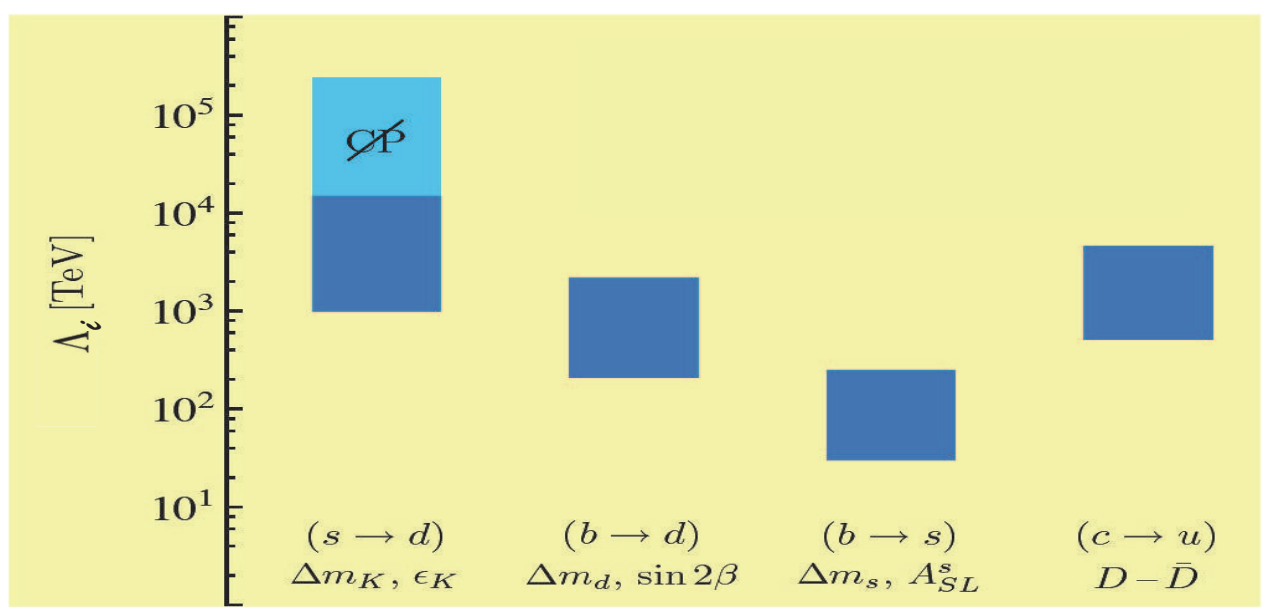

Figure 8: Range of limits on $\Lambda_{i}$ for different generic (i.e. $c_{i}=1$ ) new physics FCNC processes channels for particle antiparticle oscillation and $C P V$ measurements. Here $\varepsilon_{K}$ is the CPV parameter for the $K^{0}$, system, $\beta=\phi_{1}$, and $A_{\mathrm{SL}}^{s}$ is the limit on a $C P V$ asymmetry in semileptonic $B_{s}^{0}$ decays. (From M. Neubert's talk at EPS HEP 2011.)

\section{Search for signs of a charged Higgs}

A feature common to many BSM theories is that they have Higgs sectors that are more complicated than the single neutral Higgs scalar of the SM. For example, in SUSY models there are five Higgs scalars, including a doublet of charged Higgs particles $H^{ \pm}$, and these all have SUSY-partner higgsino fermions. The search for charged Higgs particles is a major activity of the high- $p_{T}$ LHC experiments.

Models with charged Higgs doublets are classified into three types: in Type I models, the Higgs couples to up-type and down-type quarks with equal strength; in Type II models, the couplings to up-type and down-type quarks differ by a ratio that is commonly expressed as $\tan \beta$; Type III models are all other cases. Since many versions of SUSY are Type II models, these have been the most extensively studied.

Since Higgs couplings are proportional to mass, $B$ meson decays to final states containing $\tau$ leptons are most sensitive to possible effects from charged Higgs. Figure 9(a) shows the quark-line diagram for purely leptonic $B^{+} \rightarrow \tau^{+} v_{\tau}$ decay. $^{2}$

In the SM, this decay is mediated by a virtual $W^{+}$boson that couples to the $\bar{b} u$ vertex with a strength $V_{u b} G_{F}$; the SM expression for the branching fraction is

$$
\mathscr{B}\left(B^{+} \rightarrow \tau^{+} v_{\tau}\right)=\frac{G_{F}^{2} m_{B} m_{\tau}^{2}}{8 \pi}\left(1-\frac{m_{\tau}^{2}}{m_{B}^{2}}\right) f_{B}^{2}\left|V_{u b}\right|^{2} \tau_{B}=\left(0.73_{-0.07}^{+0.12}\right) \times 10^{-4},
$$

\footnotetext{
${ }^{2}$ In this discussion, the inclusion of charge conjugate modes is implied.
} 
where $f_{B}$ is the $B^{+}$decay constant calulated by Lattice QCD and $\tau_{B}$ is the $B^{+}$meson lifetime.

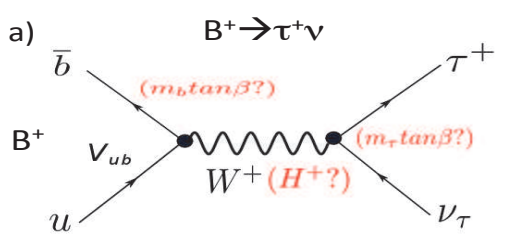

c)

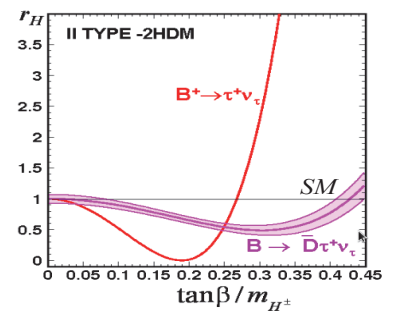

b)

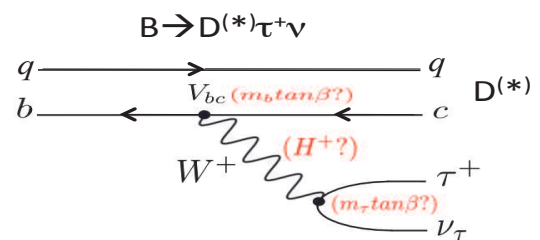

d)

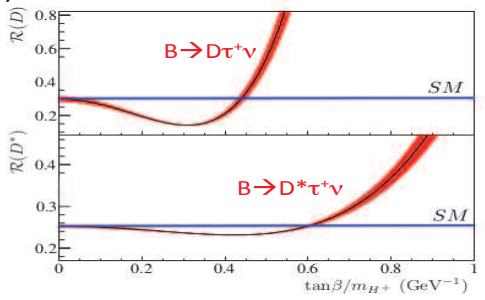

Figure 9: Quark line diagrams for $a$ ) $B^{+} \rightarrow \tau^{+} v_{\tau}$ and $\left.b\right) B \rightarrow D^{(*)} \tau^{+} v_{\tau}$. In the SM, the decays are mediated by virtual $W^{+}$bosons; in BSM theories, virtual charged Higgs bosons $\left(H^{ \pm}\right)$can also contribute. c) The ratio of the expected $B^{+} \rightarrow \tau^{+} v_{\tau}$ branching fraction and its SM value ( $\left.r_{H}\right)$ as a function of $\tan \beta / M_{H}$.d) Corresponding ratios for $B \rightarrow D \tau^{+} v_{\tau}$ (upper) and $B \rightarrow D^{*} \tau^{+} v_{\tau}$ (lower).

If there is a Type II charged Higgs with mass $m_{H}$, it can also mediate this decay and would couple to the $\bar{b} u$ vertex with a strength $m_{b} \tan \beta$ and to the $\tau \nu$ decay vertex with a strength $m_{\tau} \tan \beta$. This modifies the branching fraction by a factor [36]

$$
r_{H}=1-\frac{m_{B}^{2}}{m_{H}^{2}} \tan ^{2} \beta
$$

which is shown as a function of $\tan \beta / m_{H}$ as a red curve in Fig. 9(c).

The same charged Higgs would also modify SM expectations for semileptonic $B \rightarrow D^{(*)} \tau \nu$ decays as shown in Fig 9(b). Here the SM "predictions" are the measured branching fractions for $B \rightarrow D^{(*)} \ell^{+} v\left(\ell^{+}=\mu^{+}\right.$or $\left.e^{+}\right)$scaled by factor that reflects the reduced phase space for $\ell^{+}=\tau^{+}$. The Type II model charged-Higgs-induced modifications for the $D \tau \nu$ and $D^{*} \tau \nu$ final states are different, and given by the ratios to $D \ell^{+} v$ and $D^{*} \ell^{+} v, R_{D}$ and $R_{D^{*}}$, that are shown as a function of $\tan \beta / m_{H}$ in the upper and lower sections of Fig. 9(d), respectively.

\subsection{Experimental issues with $B \rightarrow \tau v$ and $B \rightarrow D^{(*)} \tau \nu$ measurements}

Experimentally, measurements of $B$ meson decay channels that contain a $\tau$ and a $v_{\tau}$ are challenging. Final states have at least two neutrinos and, thus, missing mass techniques commonly used to study single neutrino final states associated with $e^{-} v$ and $\mu^{-} v$ lepton pairs are not applicable. The BaBar and Belle experiments use the "tagged $B$ " technique that exploits the fact that the $B$ mesons produced in $e^{+} e^{-}$collisions at the $\Upsilon(4 S)$ are produced in $B \bar{B}$ pairs, with no accompanying particles. Thus, if one $B$ meson decay is completely reconstructed, one knows with confidence that any remaining particles in an event must be decay products from the accompanying $\bar{B}$. For the $B^{-} \rightarrow \tau^{-} \bar{v}$ measurement, Belle selects events that contain one fully reconstructed $B^{+}$meson and a single accompanying charged track, which is potentially a lepton from $\tau^{-} \rightarrow \ell^{-} v \bar{v}$ or a $\pi^{-}$from $\tau^{-} \rightarrow \pi^{-} \bar{v}$ [15]. An event display of a candidate $B^{-} \rightarrow \tau^{-} v$ decay in Belle is shown in Fig. 10(a), 
where a $B^{+} \rightarrow \bar{D}^{0} \pi^{+}$decay, with $\bar{D}^{0} \rightarrow K^{+} \pi^{-} \pi^{+} \pi^{-}$, is reconstructed in the tracking system, along with a single, well identified electron. Many other $B^{-}$decay channels produce a single track in the detector. However, these also contain other neutral particles, such as $\gamma$-rays, $\pi^{0} \mathrm{~s}, K_{L}$ mesons, etc. Usually, these particles deposit sgnificant amounts of energy in the CsI calorimeter that surrounds the Belle tracking system, covering $\sim 85 \%$ of the total $4 \pi$ solid angle. The signature for $B^{-} \rightarrow \tau^{-} \bar{v}$ is an excess of events with small excess energy deposit in the calorimeter, as shown in Fig. 10(b). Here the background level is established by studying events in which a tagged $B$ is accompanied by a meson that decays via the semileptonic $B^{-} \rightarrow D^{* 0} \ell^{-} \bar{v}$ decays, and other well understood control samples. The $B \rightarrow D^{(*)} \tau \nu$ event selection, described in Ref. [17], is similar. Since the probability for fully reconstructing the accompanying $B$ meson is very low, $\sim 0.25 \%$, this method has very low efficiency, but it is best that one can do. These measurements can only be done at an $e^{+} e^{-}$ $B$-factory operating at the $\Upsilon(4 S)$.
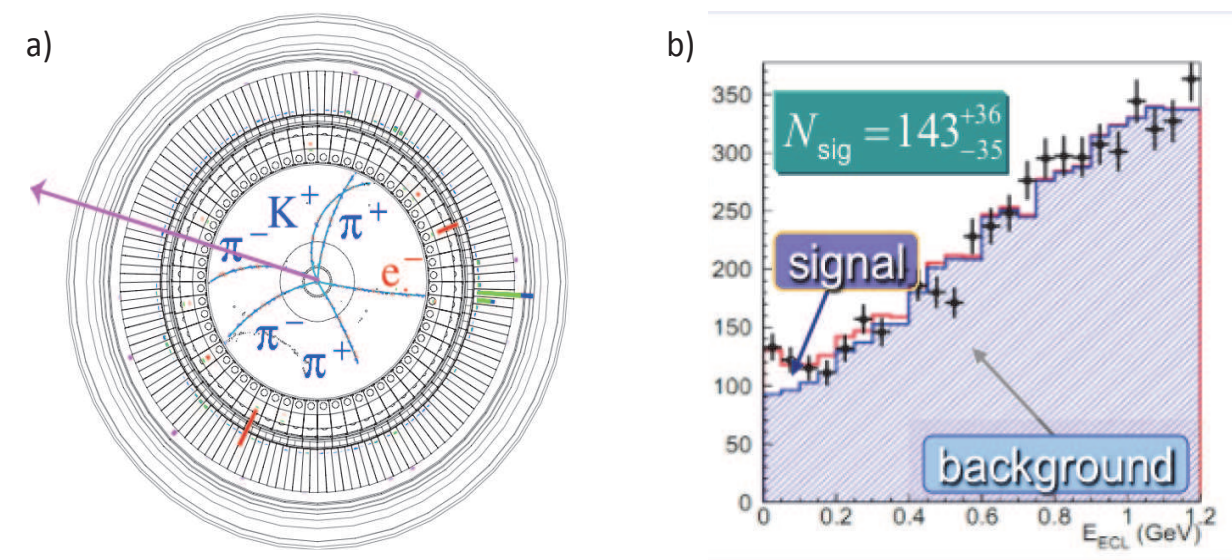

Figure 10: a) An event display of a $B^{-} \rightarrow \tau^{-} \bar{v}$ event candidate in Belle. Here a $B^{+}$is fully reconstructed as discussed in the text and the $\tau$ decays via the $\tau^{-} \rightarrow e^{-} v \bar{v}$ mode. Here the purple arrow indicates the direction of the missing momentum in the event. $b$ ) The distribution of unassigned energy in the CsI calorimeter for $B \rightarrow \tau v$ candidate events. The small excess over background below $200 \mathrm{MeV}$ is the signal for $B \rightarrow \tau \nu$.

The 2013 PDG world average $B \rightarrow \tau v$ branching fraction is $(1.05 \pm 0.25) \times 10^{-4}$ [27], which is higher than the SM value, given above in Eq. 3.1, by $\sim 1 \sigma$. Results from Belle [17] and BaBar [18] on $B \rightarrow D \tau \nu$ and $B \rightarrow D^{*} \tau \nu$ range from $1 \sigma$ to $\sim 2.5 \sigma$ above the SM expectations. These discrepancies are intriguing, but not significant enough to make any claims.

The situation is summarized in Fig. 11, where current experimental results, shown as blue bands, are superimposed on the curves indicating expectations from a Type II Higgs doublet model shown above in Fig. 9. The locations of the crossing points of the experimental bands with the red expectation curves should locate the value of $\tan \beta / m_{H}$. As can be seen in the figure, the preferred $\tan \beta / m_{H}$ values for each mode are inconsistent: $\sim 0.28$ for $\tau \nu, \sim 0.45$ for $D \tau \nu$, and $\sim 0.8$ for $D^{*} \tau \nu$. These discrepancies are undoubtably are due to the large statistical errors - there is no compelling evidence in the data for a charged Higgs to begin with. However, one could imagine a situation where, with 40 times more data as expected for BelleII, the results settle on the current central values but with error bars that are four or five times smaller and, at the same time, LHC experiments report signals for a charged Higgs. In that case, not only would the $B$ 

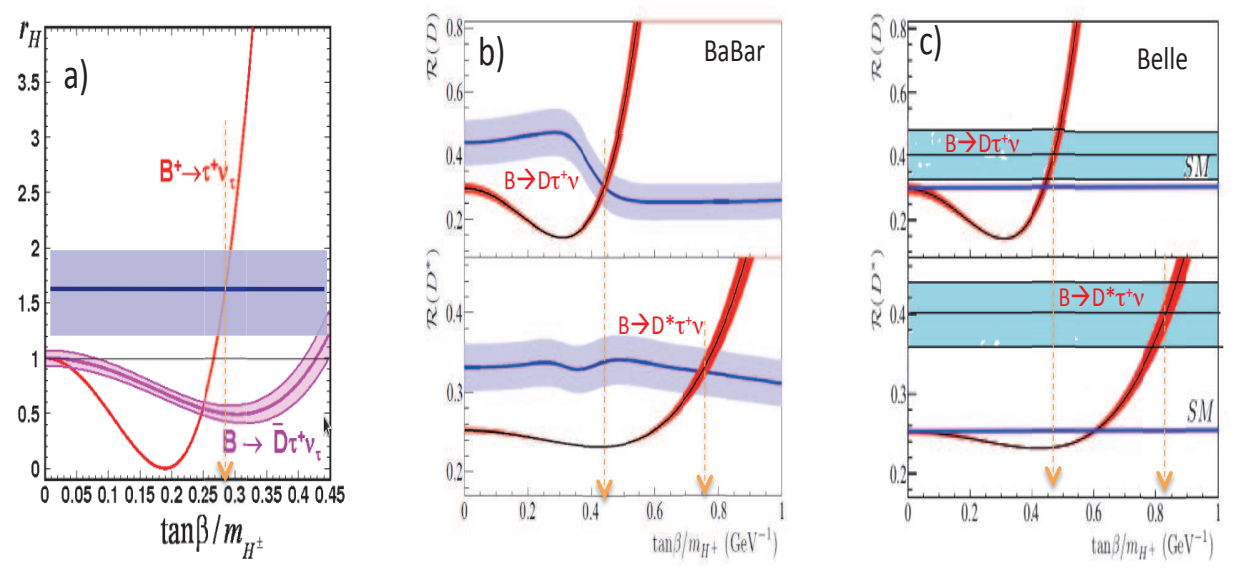

Figure 11: a) The blue band shows combined BaBar and Belle results on $B^{-} \rightarrow \tau^{-} \bar{v}$ compared with expectations from the SM (horizontal line at $r_{H}=1$ ) and the Type II Higgs doublet model. The measured value is about $1 \sigma$ above SM expectations. b) BaBar results on $B \rightarrow D \tau \nu$ (upper) and $D^{+} \tau \nu$ (lower). Here for both modes the measured values are about $2 \sigma$ above SM expectations. $c$ ) Corresponding results from Belle.

decay measurements provide additional compelling evidence for some BSM process, but also clear diagnostic evidence that rules out its interpretation as a Type II charged Higgs.

\section{Comments}

In this talk I intentionally avoided giving a shopping list of physics topics ${ }^{3}$ and, instead, tried to provide a few detailed and concrete examples of how flavor physics measurements place tight constraints on any proposed model for physics beyound the Standard Model, and also how future precision measurements of rare processes can probe for new physics at mass scales that are far above those that will ever be accessed by the LHC. I described a scenario that illustrates how flavor physics measurements will provide essential diagnostic information that can help classify the "DNA" of a charged Higgs candidate that might be observed by LHC experiments. The flavor physics measurements are an essential component of new, BSM physics physics searches.

\section{Acknowledgements}

I thank the organizers for inviting me to give this talk and congratulate them on another interesting and successful Bormio meeting. This work was supported in part by the Korean National Research Foundation (NRF) Grant No. 20110029457.

\section{References}

[1] G. Aad et al. (ATLAS Collaboration), Phys. Lett. B716, 1 (2012).

[2] S. Chatrchyan et al. (CMS Collaboration), Phys. Lett. B716, 30 (2012).

\footnotetext{
${ }^{3}$ More comprehensive coverage of the broad range of topics that are on the agenda for near-future, intensity-frontier flavor physics experiments are provided in Refs. [19] and [20], and references cited therein.
} 
[3] See, for example, K. Agashe et al., arXiv:1311.0299.

[4] See, for example, H.E. Haber and M. Schmitt Eur. Phys. J. C3, 743 (1998).

[5] See, for example, K.S. Babu, C.F Kolda and J. March-Russell Eur. Phys. J. C15, 287 (2000).

[6] See, for example, George W.S. Hou PoS ICHEP2010, 244 (2010).

[7] See, for example, Ewa Stanecka (ATLAS Collaboration) and Lars Sonnenschein (CMS) Collaboration), these proceediings.

[8] R. Aaij et al. (LHCb Collaboration) Phys. Rev. Lett. 111, 101805 (2013).

[9] Ulrich Uwer, these proceedings.

[10] R. Aaij et al. (LHCb Collaboration) New J. Phys. 15, 053021 (2013).

[11] A. Lenz and U. Nierste arXiv:1102.4274 and D. Guadagnoli and G. Isidori, Phys. Lett. B724, 63 (2013).

[12] M. Kobayashi and H. Maskawa, Prog. Theor. Phys. 49, 652 (1973).

[13] B. Aubert et al. (BaBar Collaboration) Phys. Rev. Lett. 87, 091801 (2001) and K. Abe et al. (Belle Collaboration) Phys. Rev. Lett. 87, 091802 (2001).

[14] J.P. Lees et al. (BaBar Collaboration) Phys. Rev. D 85, 112010 (2012) and Y. Nakahama et al. (Belle Collaboration) Phys. Rev. D 82, 073011 (2010).

[15] I. Adachi et al. (Belle Collaboration) Phys. Rev. lett. 110, 131801 (2013).

[16] J.P. Lees et al. (BaBar Collaboration) Phys. Rev. D 88, 031102 (2013).

[17] A. Bozek et al. (Belle Collaboration) Phys. Rev. D 82, 072005 (2010).

[18] B. Aubert et al. (BaBar Collaboration) Phys. Rev. D 79, 092002 (2009).

[19] T. Aushev et al. (BelleII Collaboration) arXiv:1002.5012.

[20] B. O'Leary et al. (SuperB Collaboration) arXiv:1008.1541.

[21] M. Gell-Mann and A. Pais, Phys. Rev. 97, 1387 (1955).

[22] J.H. Christenson, J.W. Cronin, V.L. Fitch and R. Turlay, Phys. Rev. lett. 13, 138 (1964).

[23] S.L. Glashow, J. Iliopoulos and L. Maiani, Phys. Rev. D 2, 1285 (1970).

[24] H. Albrecht et al. (ARGUS Collaboration) Phys. Lett. B192, 245 (1987).

[25] See, for example, N. Barik, P. Das, A.R. Panda and K.C. Roy, Phys. Rev. D 48, 3271 (1993).

[26] F. Abe et al. (CDF Collaboration) Phys. Rev. Lett. 74, 2626 (1995) and S. Abachi et al. (D0 Collaboration) Phys. Rev. Lett. 74, 2632 (1995).

[27] J. Beringer et al. (Particle Data Group), Phys. Rev. D 86, 010001 (2012).

[28] Y. Grossman, CERN Seminar 2007/3/27.

[29] Y. Amhis et al. (Heavy Flavor Averaging Group), arXiv:1207.1158.

[30] J. Charles et al. CKMfitter Group), Eur. Phys. J. C41, 1 (2005).

[31] Jianglei Yu, arXiv:1312.0306.

[32] Y. Nir and G. Raz, Phys. Rev. D 66, 035007 (2002). 
[33] B. Aubert et al. (BaBar Collaboration) Phys. Rev. Lett. 98, 211802 (2007) and M. Staric et al. (Belle Collaboration) Phys. Rev. Lett. 98, 211803 (2007).

[34] B. Aubert et al. (BaBar Collaboration) Phys. Rev. D 79, 072009 (2009) and I. Adachi et al. (Belle Collaboration) Phys. Rev. Lett. 108, 171802 (2012).

[35] M. Neubert, plenary talk at EPS HEP 2011, Grenoble, July 21-27. See also G. Isidori, Y. Nir and G. Perez, Ann. Rev. Nucl. Part. Sci.60, 355 (2010).

[36] W.S. Hou, Phys. Rev. D 48, 2342 (1993). 